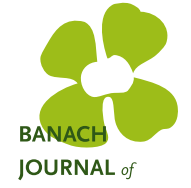

MOURNAL of ANALYSIS
Banach J. Math. Anal. 13 (2019), no. 2, 468-485

https://doi.org/10.1215/17358787-2018-0049

ISSN: $1735-8787$ (electronic)

http://projecteuclid.org/bjma

\title{
SOLID CORES AND SOLID HULLS OF WEIGHTED BERGMAN SPACES
}

\author{
JOSÉ BONET, ${ }^{1 *}$ WOLFGANG LUSKY,${ }^{2}$ and JARI TASKINEN ${ }^{3}$ \\ Communicated by M. González Órtiz
}

\begin{abstract}
We determine the solid hull for $2<p<\infty$ and the solid core for $1<p<2$ of weighted Bergman spaces $A_{\mu}^{p}, 1<p<\infty$, of analytic functions on the disk and on the whole complex plane, for a very general class of nonatomic positive bounded Borel measures $\mu$. New examples are presented. Moreover, we show that the space $A_{\mu}^{p}, 1<p<\infty$, is solid if and only if the monomials are an unconditional basis of this space.
\end{abstract}

\section{Introduction and preliminaries}

Consider $R=1$ or $R=\infty$ and $\mathbb{D}=\{z \in \mathbb{C}:|z|<1\}$. We study holomorphic functions $f: R \cdot \mathbb{D} \rightarrow \mathbb{C}$, where $R \cdot \mathbb{D}=\mathbb{D}$ if $R=1$ and $R \cdot \mathbb{D}=\mathbb{C}$ if $R=\infty$. Let $\hat{f}(k)$ be the Taylor coefficients of $f$; that is, $f(z)=\sum_{k=0}^{\infty} \hat{f}(k) z^{k}$. We take a nonatomic positive bounded Borel measure $\mu$ on $[0, R[$ such that $\mu([r, R[)>0$ for every $r>0$ and $\int_{0}^{R} r^{n} d \mu(r)<\infty$ for all $n>0$. Put, for $1 \leq p<\infty$,

$$
\|f\|_{p}=\left(\frac{1}{2 \pi} \int_{0}^{R} \int_{0}^{2 \pi}\left|f\left(r e^{i \varphi}\right)\right|^{p} d \varphi d \mu(r)\right)^{1 / p}
$$

and let

$$
A_{\mu}^{p}=\left\{f: R \cdot \mathbb{D} \rightarrow \mathbb{C}: f \text { holomorphic with }\|f\|_{p}<\infty\right\} .
$$

We call $A_{\mu}^{p}$ a weighted Bergman space.

Copyright 2019 by the Tusi Mathematical Research Group.

Received Jul. 9, 2018; Accepted Dec. 10, 2018.

First published online Feb. 26, 2019.

${ }^{*}$ Corresponding author.

2010 Mathematics Subject Classification. Primary 46E15; Secondary 46B15.

Keywords. weighted Bergman spaces, solid hulls, solid cores. 
Let $H(R \cdot \mathbb{D})$ be the space of all holomorphic functions on $R \cdot \mathbb{D}$, and let $A \subset H(R \cdot \mathbb{D})$ be a subspace containing the polynomials. We want to study the solid core

$$
s(A)=\{f \in A: g \in A \text { for all holomorphic } g \text { with }|\hat{g}(k)| \leq|\hat{f}(k)| \text { for all } k\}
$$

and the solid hull

$$
\begin{aligned}
S(A)= & \{g: R \cdot \mathbb{D} \rightarrow \mathbb{C}: g \text { holomorphic, there is } f \in A \text { with } \\
& |\hat{g}(k)| \leq|\hat{f}(k)| \text { for all } k\} .
\end{aligned}
$$

We call $A$ solid if $A=S(A)$. In the first four sections we consider $A=A_{\mu}^{p}$, while in Section 5 we include the case where $A$ consists of weighted sup-norm spaces of holomorphic functions.

The solid hull and core of spaces of analytic functions has been investigated by many authors. We refer the reader to the recent books [6] and [13] and the many references therein. For example, [6] presented a characterization of solid hulls and cores of $A_{\mu}^{p}$ where $d \mu(r)=(1-r)^{\alpha} d r$ for some $\alpha>0$ and $R=1$.

Originally, our main interest was to replace the "standard weights" $(1-r)^{\alpha}$ by weights of the form $v_{a, b}(r)=\exp \left(-a /(1-r)^{b}\right)$ for some $a>0$ and $b>0$, which are of a completely different nature and require different methods, and hence to consider $d \mu(r)=v_{a, b}(r) d r$. We wanted to extend to weighted Bergman spaces the results of [1] and [2], works which were entirely devoted to this class of weights $v_{a, b}$ in connection with weighted sup-norms. In the present article we give a characterization of solid hulls of $A_{\mu}^{p}$ if $2<p<\infty$ and solid cores of $A_{\mu}^{p}$ if $1<p<2$ in our main Theorem 2.1 for much more general $\mu$ which, under some mild additional assumptions (Corollary 3.2), results in the explicit computation of many examples including $v(r)=\exp \left(-a /(1-r)^{b}\right)$ for $R=1$ and $v(r)=\exp (-r)$ for $R=\infty$ (see Corollaries 3.4 and 3.5). Finally, Sections 4 and 5 are dedicated to Bergman spaces $A_{\mu}^{p}$ and weighted sup-norm spaces $H_{v}^{\infty}$ which are themselves solid. We give examples for this situation in connection with holomorphic functions over the complex plane and show that this can never happen for holomorphic functions over the unit disk. The main results are Theorem 4.1, which states that $A_{\mu}^{p}$ is solid if and only if the monomials $\left(z^{n}\right)_{n=0}^{\infty}$ are an unconditional basis of $A_{\mu}^{p}$, and Theorem 5.2, which ensures that $H_{v}^{\infty}$ is solid if and only if $\left(z^{n}\right)_{n=0}^{\infty}$ is a Schauder basis of the closure $H_{v}^{0}$ of the polynomials in $H_{v}^{\infty}$.

For a holomorphic $g$ and $0<r$, we define

$$
M_{p}(g, r)=\left(\frac{1}{2 \pi} \int_{0}^{2 \pi}\left|g\left(r e^{i \varphi}\right)\right|^{p} d \varphi\right)^{1 / p}
$$

and $P_{n} g(z)=\sum_{k=0}^{n} \hat{g}(k) z^{k}$. It is well known that, for $1<p<\infty$, there are universal constants $c_{p}>0$ with $M_{p}\left(P_{n} g, r\right) \leq c_{p} M_{p}(g, r)$, where $c_{p}$ does not depend on $g, n$, or $r$. Moreover, we have $\lim _{n \rightarrow \infty} M_{p}\left(g-P_{n} g, r\right)=0$. Hence, we obtain

$$
\left\|P_{n} f\right\|_{p} \leq c_{p}\|f\|_{p} \quad \text { for all } f \in A_{\mu}^{p} \text { and all } n \quad \text { and } \quad \lim _{n \rightarrow \infty}\left\|f-P_{n} f\right\|_{p}=0 .
$$


In particular, we see that the monomials $z \mapsto z^{n}, n=0,1,2, \ldots$ form a Schauder basis of $A_{\mu}^{p}$ if $1<p<\infty$. (Details can be found in [4] and [14].) In the rest of the article $[r]$ denotes the largest integer less than or equal to $r>0$.

\section{Main general result}

The main result of this section is Theorem 2.1 below. There are relevant earlier, related works. For example, in Theorem 4.1 of [12], Pavlović established a useful norm in blocks for certain weighted Bergman spaces. (See also earlier work by Mateljević and Pavlović [11].)

Theorem 2.1. Assume that there are constants $d_{1}, d_{2}>0$, and $\omega_{n}>0, n=$ $1,2, \ldots$, numbers $0 \leq l_{1}<l_{2}<\cdots$, and radii $s_{1}<s_{2}<\cdots$ such that, for every $f \in A_{\mu}^{p}$,

$$
d_{1}\|f\|_{p} \leq\left(\sum_{n=1}^{\infty} \omega_{n}^{p} M_{p}^{p}\left(\left(P_{\left[l_{n+1}\right]}-P_{\left[l_{n}\right]}\right) f, s_{n}\right)\right)^{1 / p} \leq d_{2}\|f\|_{p} .
$$

(a) If $2<p<\infty$, then

$$
\begin{aligned}
S\left(A_{\mu}^{p}\right)= & \{g: R \cdot \mathbb{D} \rightarrow \mathbb{C}: \\
& \left.g \text { holomorphic with } \sum_{n=1}^{\infty} \omega_{n}^{p}\left(\sum_{k=\left[l_{n}\right]+1}^{\left[l_{n+1}\right]}|\hat{g}(k)|^{2} s_{n}^{2 k}\right)^{p / 2}<\infty\right\} .
\end{aligned}
$$

(b) If $1<p<2$, then

$$
\begin{aligned}
s\left(A_{\mu}^{p}\right)= & \{g: R \cdot \mathbb{D} \rightarrow \mathbb{C}: \\
& \left.g \text { holomorphic with } \sum_{n=1}^{\infty} \omega_{n}^{p}\left(\sum_{k=\left[l_{n}\right]+1}^{\left[l_{n+1}\right]}|\hat{g}(k)|^{2} s_{n}^{2 k}\right)^{p / 2}<\infty\right\} .
\end{aligned}
$$

Theorem 2.1 is proved below. Before presenting the proof, we point out that condition (2.1) can be realized for any given $\mu$. Indeed, fix $\beta>16 \cdot 3^{p-1}\left(1+2^{p}\right) c_{p}^{p}+2$, and use induction to obtain $0=l_{1}<l_{2}<l_{3}<\cdots$ and $0 \leq s_{1}<s_{2}<\cdots<R$ with

$$
\int_{0}^{s_{n}} r^{l_{n} p} d \mu=\beta \int_{s_{n}}^{R} r^{l_{n} p} d \mu \quad \text { and } \quad \int_{0}^{s_{n}} r^{l_{n+1} p} d \mu=\frac{1}{\beta} \int_{s_{n}}^{R} r^{l_{n+1} p} d \mu .
$$

Instead of starting with $n=1$, we can just as well start the induction with $n=n_{0}$, for example, for some $n_{0} \geq 0$ (with $l_{1}=0$ and arbitrary $s_{1}$ ) and restrict the preceding relations to all $n \geq n_{0}$. Moreover, put

$$
\omega_{n}=\left(\int_{0}^{s_{n}}\left(\frac{r}{s_{n}}\right)^{l_{n} p} d \mu+\int_{s_{n}}^{R}\left(\frac{r}{s_{n}}\right)^{l_{n+1} p} d \mu\right)^{1 / p} .
$$

Then there are constants $d_{1}, d_{2}>0$ such that, for every $f \in A_{\mu}^{p}$,

$$
d_{1}\|f\|_{p} \leq\left(\sum_{n=1}^{\infty} \omega_{n}^{p} M_{p}^{p}\left(\left(P_{\left[l_{n+1}\right]}-P_{\left[l_{n}\right]}\right) f, s_{n}\right)\right)^{1 / p} \leq d_{2}\|f\|_{p} .
$$


This was shown in [5] for $p=1$ and in [10] for $1<p<\infty$ and $R=1$, but with some slight modifications the proofs carry over to the case $R=\infty$.

Example 2.2.

(i) Let $d \mu(r)=d r$ where $R=1$. Then we obtain

$l_{n}=\frac{1}{p}\left(a^{n-1}-1\right) \quad$ and $\quad s_{n}=\left(\frac{\beta}{\beta+1}\right)^{a^{1-n}} \quad$ where $a=\frac{\log (\beta+1)}{\log (1+\beta)-\log (\beta)}$.

This can be easily verified using the definition (starting with $n=0$ ) and induction.

(ii) Let $d \mu(r)=r^{\alpha} d r$ for some $\alpha>0$ and $R=1$. With example (i) and $l_{n} p+\alpha=\left(a^{n-1}-1\right)$, where $a$ is the number in (i), we obtain

$$
l_{n}=\frac{1}{p}\left(a^{n-1}-1\right)-\frac{\alpha}{p} \quad \text { and } \quad s_{n}=\left(\frac{\beta}{\beta+1}\right)^{a^{1-n}}
$$

for $n \geq 2$ with $l_{1}=0$ and $s_{1}=\beta /(\beta+1)$.

Now we turn to the proof of Theorem 2.1. Let $f: R \cdot \mathbb{D} \rightarrow \mathbb{C}$ be holomorphic. Recall that $\hat{f}(n) r^{n}=\frac{1}{2 \pi} \int_{0}^{2 \pi} f\left(r e^{i \varphi}\right) e^{-i n \varphi} d \varphi$ for each $0<r<R$ and each $n=$ $0,1,2, \ldots$ For $g\left(r e^{i \varphi}\right)=r^{n(p-1)} e^{-i n \varphi} /\left(\int_{0}^{R} r^{n p} d \mu\right)^{1-1 / p}$, we have

$$
|\hat{f}(n)|\left(\int_{0}^{R} r^{n p} d \mu\right)^{1 / p}=\frac{1}{2 \pi}\left|\int_{0}^{R} \int_{0}^{2 \pi} f\left(r e^{i \varphi}\right) g\left(r e^{i \varphi}\right) d \varphi d \mu\right| \leq\|f\|_{p} .
$$

In the following, we make use of the Khintchine inequality (see [7, Theorem 2.b.3.]); that is, for arbitrary $b_{k}$ and $n$ we have

$$
A_{p}\left(\sum_{k=1}^{n}\left|b_{k}\right|^{2}\right)^{1 / 2} \leq\left(\frac{1}{2^{n}} \sum_{\theta_{k}= \pm 1}\left|\sum_{k=1}^{n} b_{k} \theta_{k}\right|^{p}\right)^{1 / p} \leq B_{p}\left(\sum_{k=1}^{n}\left|b_{k}\right|^{2}\right)^{1 / 2},
$$

where $A_{p}, B_{p}$ are universal constants not depending on $n$. (The summation in the central expression runs over the $2^{n}$ different possibilities of the change of signs.)

Conclusion of the proof of Theorem 2.1. For a holomorphic function $g$ put

$$
\alpha(g)=\left(\sum_{n=1}^{\infty} \omega_{n}^{p} M_{p}^{p}\left(\left(P_{\left[l_{n+1}\right]}-P_{\left[l_{n}\right]}\right) f, s_{n}\right)\right)^{1 / p} .
$$

As assumed, $\alpha(\cdot)$ is equivalent to $\|\cdot\|_{p}$. Moreover, let

$$
\gamma(g)=\left(\sum_{n=1}^{\infty} \omega_{n}^{p}\left(\sum_{k=\left[l_{n}\right]+1}^{\left[l_{n+1}\right]}|\hat{g}(k)|^{2} s_{n}^{2 k}\right)^{p / 2}\right)^{1 / p},
$$

and let $V=\{g: R \cdot \mathbb{D} \rightarrow \mathbb{C}: g$ holomorphic with $\gamma(g)<\infty\}$. Recall Parseval's identity, which implies that

$$
M_{2}^{2}\left(\left(P_{\left[l_{n+1}\right]}-P_{\left[l_{n}\right]}\right) f, s_{n}\right)=\sum_{k=\left[l_{n}\right]+1}^{\left[l_{n+1}\right]}|\hat{g}(k)|^{2} s_{n}^{2 k} .
$$


Proof of (a). Let $g \in S\left(A_{\mu}^{p}\right)$. Then there is $f \in A_{\mu}^{p}$ with $|\hat{g}(k)| \leq|\hat{f}(k)|$ for all $k$. If $2<p<\infty$, then

$$
\gamma(g) \leq \gamma(f) \leq \alpha(f) \leq d_{2}\|f\|_{p}<\infty
$$

Hence $g \in V$.

Now let $g \in V$. Put $\Delta_{n}=\{+1,-1\}^{\left[l_{n+1}\right]-\left[l_{n}\right]}$. For $\Theta_{n}=\left(\theta_{\left[l_{n}\right]+1}, \ldots, \theta_{\left[l_{n+1}\right]}\right) \in$ $\Delta_{n}$, put

$$
g_{\Theta_{n}}(\varphi)=\sum_{k=\left[l_{n}\right]+1}^{\left[l_{n+1}\right]} \theta_{k} \hat{g}(k) s_{n}^{k} e^{i k \varphi} \quad \text { and } \quad g_{n}(\varphi)=\sum_{k=\left[l_{n}\right]+1}^{\left[l_{n+1}\right]} \hat{g}(k) s_{n}^{k} e^{i k \varphi}
$$

Let $\tilde{\Theta}_{n}$ be such that

$$
M_{p}\left(g_{\tilde{\Theta}_{n}}, s_{n}\right) \leq\left(\frac{1}{2^{\left[l_{n+1}\right]-\left[l_{n}\right]}} \sum_{\Theta_{n} \in \Delta_{n}} M_{p}^{p}\left(g_{\Theta_{n}}, s_{n}\right)\right)^{1 / p} .
$$

The Khintchine inequality yields

$$
M_{p}\left(g_{\tilde{\Theta}_{n}}, s_{n}\right) \leq B_{p} M_{2}\left(g_{n}, s_{n}\right)
$$

Put $h=\sum_{n} g_{\tilde{\Theta}_{n}}$. Then, by the preceding estimates,

$$
d_{1}\|h\|_{p} \leq \alpha(h) \leq B_{p} \gamma(g)<\infty
$$

Hence $h \in A_{\mu}^{p}$. Since by definition $|\hat{h}(k)|=|\hat{g}(k)|$ for all $k$, we obtain $g \in S\left(A_{\mu}^{p}\right)$.

Proof of (b). We retain the preceding notation. Let $g \in V$, and let $f: R \cdot \mathbb{D} \rightarrow \mathbb{C}$ be holomorphic with $|\hat{f}(k)| \leq|\hat{g}(k)|$ for all $k$. Then

$$
d_{1}\|f\|_{p} \leq \alpha(f) \leq \gamma(f) \leq \gamma(g)<\infty
$$

This implies that $f \in A_{\mu}^{p}$ and hence $g \in s\left(A_{\mu}^{p}\right)$.

Now let $g \in s\left(A_{\mu}^{p}\right)$. Let $\tilde{\Theta}_{n} \in \Delta_{n}$ be such that

$$
\left(\frac{1}{2^{\left[l_{n+1}\right]-\left[l_{n}\right]}} \sum_{\Theta_{n} \in \Delta_{n}} M_{p}^{p}\left(g_{\Theta_{n}}, s_{n}\right)\right)^{1 / p} \leq M_{p}\left(g_{\tilde{\Theta}_{n}}, s_{n}\right) .
$$

Put $h=\sum_{n} g_{\tilde{\Theta}_{n}}$. Then we obtain $|\hat{h}(k)|=|\hat{g}(k)|$ for all $k$. Hence $h \in A_{\mu}^{p}$. The Khintchine inequality together with the choice of $\tilde{\Theta}_{n}$ yields

$$
\gamma(g)=\gamma(h) \leq A_{p}^{-1} \alpha(h) \leq d_{2} A_{p}^{-1}\|h\|_{p}<\infty .
$$

We conclude that $g \in V$. 


\section{Main examples}

Quite often it is very difficult to compute the parameters $l_{n}$ and $s_{n}$ in (2.2). Therefore, it is worthwhile to consider special cases which yield an equivalent representation of the norm $\|\cdot\|_{p}$ satisfying (2.1) and which are easier to compute and cover many examples. To this end, let $v:[0, R[\rightarrow] 0, \infty[$ be a weight function; that is, let $v$ be continuous, decreasing, and let it satisfy

$$
\lim _{r \rightarrow R} v(r)=0 \quad \text { and } \quad \sup _{r} r^{n} v(r)<\infty \quad \text { for all } n>0 .
$$

Moreover, let $\nu$ be a nonatomic positive Borel measure on $[0, R[$ such that $\nu\left(\left[r, R[)>0\right.\right.$ for every $r>0$ and such that $\int_{0}^{R} r^{n} v(r) d \nu(r)<\infty$ for every $n \geq 0$. Put, for $1 \leq p<\infty$,

$$
\|f\|_{p}=\left(\int_{0}^{R} M_{p}^{p}(f, r) v(r) d \nu(r)\right)^{1 / p} .
$$

Here we consider $A_{\mu}^{p}$ with $d \mu(r)=v(r) d \nu(r)$. Actually, one can relax the conditions on $v$ somewhat. It suffices to require that $v$ be decreasing on $\left[r_{0}, R[\right.$ for some $\left.r_{0} \in\right] 0, R\left[\right.$. This follows from the fact that, for $d \tilde{\mu}=1_{\left[r_{0}, R[\right.} d \mu$, the $L_{p}$-norms with respect to $\mu$ and $\tilde{\mu}$ are equivalent. Indeed, using the fact that $M_{p}(f, r)$ is increasing with respect to $r$ for holomorphic functions $f$, we see that

$$
\int_{r_{0}}^{R} M_{p}^{p}(f, r) d \mu(r) \leq \int_{0}^{R} M_{p}^{p}(f, r) d \mu(r) \leq\left(1+\frac{\mu\left(\left[r_{0}, R[)\right.\right.}{\mu([0, R[)}\right) \int_{r_{0}}^{R} M_{p}^{p}(f, r) d \mu(r) .
$$

For any $n>0$, let $r_{n} \in\left[0, R\right.$ [ be a point where the function $r \mapsto r^{n} v(r)$ attains its global maximum. It is easily seen that $r_{m}<r_{n}$ if $m<n$. In the following, we assume that

$$
\begin{aligned}
& r_{n} \text { is the unique global maximum of } r^{n} v(r) \text { for all } n \\
& \text { and there are no further local maxima. }
\end{aligned}
$$

For example, this is the case if $v$ is differentiable and $v^{\prime} / v$ is injective. Assumption (3.1) implies that $r^{n} v(r)$ is decreasing for $r \geq r_{n}$. Moreover, we assume that $v$ satisfies the following.

Condition $\left(b_{0}\right)$. There are numbers $1<b<K$ and $m_{1}<m_{2}<\cdots$ with $\lim _{n \rightarrow \infty} m_{n}=\infty$ such that

$$
b \leq\left(\frac{r_{m_{n}}}{r_{m_{n+1}}}\right)^{m_{n}} \frac{v\left(r_{m_{n}}\right)}{v\left(r_{m_{n+1}}\right)},\left(\frac{r_{m_{n+1}}}{r_{m_{n}}}\right)^{m_{n+1}} \frac{v\left(r_{m_{n+1}}\right)}{v\left(r_{m_{n}}\right)} \leq K .
$$

Condition $\left(b_{0}\right)$ is exactly the same as condition (b) in [1], except that the treatment of weighted Banach spaces of analytic functions with sup-norms requires $2<b<K$. We refer the reader to [1] and [9] for more information and examples related to these conditions.

We take the parameters of condition $\left(b_{0}\right)$ and put

$$
I_{n}=\nu\left(\left[r_{m_{n}}, r_{m_{n+1}}\right]\right),
$$


and we assume that

$$
I_{n}<\infty \quad \text { for all } n \quad \text { and } \quad \limsup _{n \rightarrow \infty} \frac{I_{n}}{\min \left(I_{n-1}, I_{n+1}\right)}<b .
$$

Theorem 3.1. Let $1<p<\infty$. Assume that $v$ satisfies condition ( $\left.b_{0}\right)$ with (3.1) and (3.2). Then there are constants $d_{1}, d_{2}>0$ with

$$
d_{1}\|f\|_{p} \leq\left(\sum_{n=1}^{\infty} M_{p}^{p}\left(\left(P_{\left[m_{n+1} / p\right]}-P_{\left[m_{n} / p\right]}\right) f, r_{m_{n}}\right) v\left(r_{m_{n}}\right) I_{n}\right)^{1 / p} \leq d_{2}\|f\|_{p}
$$

for all $f \in A_{\mu}^{p}$.

In view of (2.1), we can apply Theorem 2.1 with the preceding $l_{n}=m_{n} / p$, $\omega_{n}^{p}=v\left(r_{m_{n}}\right) I_{n}$, and $s_{n}=r_{m_{n}}$.

Corollary 3.2. Let $d \mu=v d \nu$.

(a) If $2<p<\infty$, then

$$
\begin{aligned}
S\left(A_{\mu}^{p}\right)= & \{g: R \cdot \mathbb{D} \rightarrow \mathbb{C}: g \text { holomorphic with } \\
& \left.\sum_{n=1}^{\infty} v\left(r_{m_{n}}\right) I_{n}\left(\sum_{k=\left[m_{n} / p\right]+1}^{\left[m_{n+1} / p\right]}|\hat{g}(k)|^{2} r_{m_{n}}^{2 k}\right)^{p / 2}<\infty\right\} .
\end{aligned}
$$

(b) If $1<p<2$, then

$$
\begin{aligned}
s\left(A_{\mu}^{p}\right)= & \{g: R \cdot \mathbb{D} \rightarrow \mathbb{C}: g \text { holomorphic with } \\
& \left.\sum_{n=1}^{\infty} v\left(r_{m_{n}}\right) I_{n}\left(\sum_{k=\left[m_{n} / p\right]+1}^{\left[m_{n+1} / p\right]}|\hat{g}(k)|^{2} r_{m_{n}}^{2 k}\right)^{p / 2}<\infty\right\} .
\end{aligned}
$$

Before we prove Theorem 3.1, we present the following examples. They are concrete cases to which Corollary 3.2 applies, thus permitting us to calculate explicitly all the parameters which appear in the solid hull and solid core.

Example 3.3. (i) $R=1$ and $d \mu(r)=\exp \left(-\alpha /(1-r)^{\beta}\right) d r$ for some $\alpha, \beta>0$.

We take $v(r)=\exp \left(-\alpha /(1-r)^{\beta}\right)$ and $d \nu(r)=d r$. The weight $v$ satisfies condition $\left(b_{0}\right)$ with

$$
m_{n}=\beta\left(\frac{\beta}{\alpha}\right)^{1 / \beta} n^{2+2 / \beta}-\beta n^{2} \quad \text { and } \quad r_{m_{n}}=1-\left(\frac{\alpha}{\beta}\right)^{1 / \beta} \frac{1}{n^{2 / \beta}}
$$

and $b=e^{1}$ (see [1], Theorem 3.1.) Here $I_{n}=(\alpha / \beta)^{1 / \beta}\left(n^{-2 / \beta}-(n+1)^{-2 / \beta}\right)$. Hence

$$
\lim _{n \rightarrow \infty} \frac{I_{n}}{\min \left(I_{n-1}, I_{n+1}\right)}=1 \text {. }
$$

This shows that (3.2) is satisfied. We note that (3.1) holds, too, according to [1]. So we can apply Corollary 3.2.

(ii) $R=1$ and $d \mu(r)=(1-\log (1-r))^{-1} d r$. 
Here we take

$$
v(r)=1-r \quad \text { and } \quad d \nu(r)=\frac{d r}{(1-r)(1-\log (1-r))} .
$$

Note that $r_{m}=1-1 /(m+1)$ is the only zero of the derivative of $r^{m} v(r)$. Hence (3.1) is satisfied. If we take $m_{n}=9^{n}$ and hence $r_{m_{n}}=1-1 /\left(9^{n}+1\right)$, then a simple calculation reveals that $v$ satisfies condition $\left(b_{0}\right)$ with $b=3$. We obtain

$$
I_{n}=\int_{r_{m_{n}}}^{r_{m_{n+1}}} d \nu=\log \left(\frac{1+\log \left(9^{n+1}+1\right)}{1+\log \left(9^{n}+1\right)}\right)
$$

from which we infer $\lim _{n \rightarrow \infty} I_{n} / \min \left(I_{n-1}, I_{n+1}\right)=1$. This implies (3.2).

(iii) $R=\infty$ and $d \mu(r)=e^{-r} d r$.

Here we take $v(r)=e^{-r}, d \nu(r)=d r$. Note that $r_{m}=m$ is the only zero of the derivative of $r^{m} v(r)$. Hence (3.1) is satisfied. Put

$$
m_{1}=1 \quad \text { and } \quad m_{n+1}=m_{n}+2 \sqrt{m_{n}}, \quad n=1,2 \ldots, \text { and } r_{m_{n}}=m_{n} .
$$

A simple calculation yields, with

$$
\begin{aligned}
& -x-\frac{1}{2}\left(\frac{x}{1-x}\right)^{2} \leq \log (1-x) \leq-x \quad \text { if } 0<x<1 \\
& \exp \left(\frac{4 \sqrt{m}}{\sqrt{m}+2}-2\right) \leq\left(\frac{r_{m_{n}}}{r_{m_{n+1}}}\right)^{m_{n}} \frac{v\left(r_{m_{n}}\right)}{v\left(r_{m_{n+1}}\right)} \\
& =\exp \left(m \log \left(1-\frac{2}{\sqrt{m}+2}\right)+2 \sqrt{m}\right) \leq \exp \left(\frac{4 \sqrt{m}}{\sqrt{m}+2}\right) .
\end{aligned}
$$

Similarly, with

$$
\begin{aligned}
& x-\frac{x^{2}}{2} \leq \log (1+x) \leq x \text { for } 0<x<1 \\
& \exp \left(4-2\left(1+\frac{2}{\sqrt{m}}\right)\right) \leq \exp \left((m+2 \sqrt{m}) \log \left(1+\frac{2}{\sqrt{m}}\right)-2 \sqrt{m}\right) \\
& =\left(\frac{r_{m_{n+1}}}{r_{m_{n}}}\right)^{m_{n+1}} \frac{v\left(r_{m_{n+1}}\right)}{v\left(r_{m_{n}}\right)} \leq e^{4} .
\end{aligned}
$$

This shows that condition $\left(b_{0}\right)$ holds. Moreover, we easily obtain

$$
I_{n}=2 \sqrt{m_{n}} \quad \text { and } \quad \lim _{n \rightarrow \infty} \frac{I_{n}}{\min \left(I_{n-1}, I_{n+1}\right)}=1
$$

which yields (3.2). Observe that in this case we can take $m_{n}=n^{2}$ (see Theorem 3.1 in [3]). This fact is not surprising, since one can easily prove by induction that our selection of $m_{n}$ above satisfies $(n-1)^{2} \leq m_{n} \leq n^{2}$ for each $n$.

Corollary 3.4. Let $R=1$ and $d \mu(r)=\exp (-1 /(1-r)) d r$.

(a) If $2<p<\infty$, then

$$
S\left(A_{\mu}^{p}\right)=\left\{g \in H(\mathbb{D}): \sum_{n=1}^{\infty} e^{-n^{2}}\left(\frac{1}{n^{3}}\right)\left(\sum_{k=\left[n^{4} / p\right]+1}^{\left[(n+1)^{4} / p\right]}|\hat{g}(k)|^{2}\left(1-\frac{1}{n^{2}}\right)^{2 k}\right)^{p / 2}<\infty\right\} .
$$


(b) If $1<p<2$, then

$$
s\left(A_{\mu}^{p}\right)=\left\{g \in H(\mathbb{D}): \sum_{n=1}^{\infty} e^{-n^{2}}\left(\frac{1}{n^{3}}\right)\left(\sum_{k=\left[n^{4} / p\right]+1}^{\left[(n+1)^{4} / p\right]}|\hat{g}(k)|^{2}\left(1-\frac{1}{n^{2}}\right)^{2 k}\right)^{p / 2}<\infty\right\} .
$$

Proof. Example 3.3(i) and Corollary 3.2 yield, with $\alpha=\beta=1$ and $m_{n}=n^{4}-n^{2}$, $S\left(A_{\mu}^{p}\right)=\{g \in H(\mathbb{D}):$

$$
\left.\sum_{n=1}^{\infty} e^{-n^{2}}\left(\frac{1}{n^{2}}-\frac{1}{(n+1)^{2}}\right)\left(\sum_{k=\left[\left(n^{4}-n^{2}\right) / p\right]+1}^{\left[\left((n+1)^{4}-(n+1)^{2}\right) / p\right]}|\hat{g}(k)|^{2}\left(1-\frac{1}{n^{2}}\right)^{2 k}\right)^{p / 2}<\infty\right\}
$$

if $2<p<\infty$ and

$$
\begin{aligned}
s\left(A_{\mu}^{p}\right)= & \{g \in H(\mathbb{D}): \\
& \left.\sum_{n=1}^{\infty} e^{-n^{2}}\left(\frac{1}{n^{2}}-\frac{1}{(n+1)^{2}}\right)\left(\sum_{k=\left[\left(n^{4}-n^{2}\right) / p\right]+1}^{\left[\left((n+1)^{4}-(n+1)^{2}\right) / p\right]}|\hat{g}(k)|^{2}\left(1-\frac{1}{n^{2}}\right)^{2 k}\right)^{p / 2}<\infty\right\}
\end{aligned}
$$

if $1<p<2$. If we let $k$ run, in the preceding summations, from $\left[n^{4} / p\right]+1$ to $\left[(n+1)^{4} / p\right]$ instead, then we obtain conditions which are equivalent to the preceding ones and hence characterize again $S\left(A_{\mu}^{p}\right)$ and $s\left(A_{\mu}^{p}\right)$. This follows from

$$
n^{4}-n^{2} \leq n^{4} \leq(n+1)^{4}-(n+1)^{2} \text { for all } n .
$$

(Compare this with Lemma 3.2. and Example 3.3(i) in [1].) Then, finally, Corollary 3.4 follows from

$$
\left(\frac{1}{2}\right) \frac{1}{n^{3}} \leq \frac{1}{n^{2}}-\frac{1}{(n+1)^{2}} \leq \frac{2}{n^{3}} \text { for all } n .
$$

Corollary 3.5. Let $R=\infty$ and $d \mu(r)=e^{-r} d r$.

(a) If $2<p<\infty$, then

$$
S\left(A_{\mu}^{p}\right)=\left\{g \in H(\mathbb{C}): \sum_{n=1}^{\infty} e^{-n^{2}} 2 n\left(\sum_{k=\left[n^{2} / p\right]+1}^{\left[(n+1)^{2} / p\right]}|\hat{g}(k)|^{2} n^{2 k}\right)^{p / 2}<\infty\right\} .
$$

(b) If $1<p<2$, then

$$
s\left(A_{\mu}^{p}\right)=\left\{g \in H(\mathbb{C}): \sum_{n=1}^{\infty} e^{-n^{2}} 2 n\left(\sum_{k=\left[n^{2} / p\right]+1}^{\left[(n+1)^{2} / p\right]}|\hat{g}(k)|^{2} n^{2 k}\right)^{p / 2}<\infty\right\} .
$$

Proof. This is a consequence of Example 3.3(iii) and Corollary 3.2.

Lemma 3.6. Let $1 \leq p<\infty$, let $0<r<s$, and let $f(z)=\sum_{m \leq j \leq n} \alpha_{j} z^{j}$ for some $\alpha_{j}$ and $0 \leq m<n$. Then we have

$$
\text { (i) } M_{p}(f, r) \leq\left(\frac{r}{s}\right)^{m} M_{p}(f, s)
$$


and

$$
\text { (ii) } M_{p}(f, s) \leq\left(\frac{s}{r}\right)^{n} M_{p}(f, r) \text {. }
$$

Proof. Part (i) follows from the fact that, for holomorphic $f$, the function $M_{p}(f, \cdot)$ is increasing in $r$, while part (ii) is Lemma 3.1(i) of [8].

Now consider $1<p<\infty$, and let $m_{n}, I_{n}$ satisfy condition $\left(b_{0}\right)$, (3.1), and (3.2).

Lemma 3.7. Fix $k, n$, and $r_{m_{k}} \leq r \leq r_{m_{k+1}}$. Then we have

$$
\text { (i) }\left(\frac{r}{r_{m_{n}}}\right)^{m_{n}} \frac{v(r)}{v\left(r_{m_{n}}\right)} \leq\left(\frac{1}{b}\right)^{n-k-1} \quad \text { if } k<n
$$

and

$$
\text { (ii) }\left(\frac{r}{r_{m_{n}}}\right)^{m_{n+1}} \frac{v(r)}{v\left(r_{m_{n}}\right)} \leq K\left(\frac{1}{b}\right)^{k-n-1} \quad \text { if } k \geq n \text {. }
$$

Proof. If $k<n$, then we have

$$
\begin{aligned}
& \left(\frac{r}{r_{m_{n}}}\right)^{m_{n}} \frac{v(r)}{v\left(r_{m_{n}}\right)} \\
& \quad=\left(\frac{r}{r_{m_{k+1}}}\right)^{m_{n}} \frac{v(r)}{v\left(r_{m_{k+1}}\right)}\left(\frac{r_{m_{k+1}}}{r_{m_{k+2}}}\right)^{m_{n}} \frac{v\left(r_{m_{k+1}}\right)}{v\left(r_{m_{k+2}}\right)} \ldots\left(\frac{r_{m_{n-1}}}{r_{m_{n}}}\right)^{m_{n}} \frac{v\left(r_{m_{n-1}}\right)}{v\left(r_{m_{n}}\right)} \\
& \quad \leq\left(\frac{r}{r_{m_{k+1}}}\right)^{m_{k+1}} \frac{v(r)}{v\left(r_{m_{k+1}}\right)}\left(\frac{r_{m_{k+1}}}{r_{m_{k+2}}}\right)^{m_{k+2}} \frac{v\left(r_{m_{k+1}}\right)}{v\left(r_{m_{k+2}}\right)} \ldots\left(\frac{r_{m_{n-1}}}{r_{m_{n}}}\right)^{m_{n}} \frac{v\left(r_{m_{n-1}}\right)}{v\left(r_{m_{n}}\right)} \\
& \quad \leq\left(\frac{1}{b}\right)^{n-k-1} .
\end{aligned}
$$

If $k \geq n+1$, then we have

$$
\begin{aligned}
& \left(\frac{r}{r_{m_{n}}}\right)^{m_{n+1}} \frac{v(r)}{v\left(r_{m_{n}}\right)} \\
& \quad=\left(\frac{r}{r_{m_{k}}}\right)^{m_{n+1}} \frac{v(r)}{v\left(r_{m_{k}}\right)}\left(\frac{r_{m_{k}}}{r_{m_{k-1}}}\right)^{m_{n+1}} \frac{v\left(r_{m_{k}}\right)}{v\left(r_{m_{k-1}}\right)} \ldots\left(\frac{r_{m_{n+1}}}{r_{m_{n}}}\right)^{m_{n+1}} \frac{v\left(r_{m_{n+1}}\right)}{v\left(r_{m_{n}}\right)} \\
& \quad \leq\left(\frac{r}{r_{m_{k}}}\right)^{m_{k}} \frac{v(r)}{v\left(r_{m_{k}}\right)}\left(\frac{r_{m_{k}}}{r_{m_{k-1}}}\right)^{m_{k-1}} \frac{v\left(r_{m_{k}}\right)}{v\left(r_{m_{k-1}}\right)} \ldots\left(\frac{r_{m_{n+2}}}{r_{m_{n+1}}}\right)^{m_{n+1}} \frac{v\left(r_{m_{n-1}}\right)}{v\left(r_{m_{n}}\right)} K \\
& \quad \leq K\left(\frac{1}{b}\right)^{k-n-1} .
\end{aligned}
$$

Similarly, for $k=n$,

$$
\left(\frac{r}{r_{m_{n}}}\right)^{m_{n+1}} \frac{v(r)}{v\left(r_{m_{n}}\right)} \leq\left(\frac{r_{m_{n+1}}}{r_{m_{n}}}\right)^{m_{n+1}} \frac{v\left(r_{m_{n+1}}\right)}{v\left(r_{m_{n}}\right)} \leq K .
$$

Now fix $k_{0}>0$ and $0<\rho<b$ such that

$$
\frac{I_{n}}{\min \left(I_{n-1}, I_{n+1}\right)} \leq \rho \quad \text { if } k \geq k_{0} .
$$


Corollary 3.8. Let $f_{n}(z)=\sum_{m_{n} / p \leq j<m_{n+1} / p} \alpha_{j} z^{j}$, where $n \geq k_{0}$. Then, for any $k \geq k_{0}$ we have

$$
\int_{r_{m_{k}}}^{r_{m_{k+1}}} M_{p}^{p}\left(f_{n}, r\right) v(r) d \nu(r) \leq c\left(\frac{\rho}{b}\right)^{|n-k|} M_{p}^{p}\left(f_{n}, r_{m_{n}}\right) v\left(r_{m_{n}}\right) I_{n} .
$$

Here $c>0$ is a universal constant independent of $k, n, f_{n}$.

Proof. First let $k<n$. Then Lemmas 3.6(i) and 3.7(i) imply that

$$
\begin{aligned}
\int_{r_{m_{k}}}^{r_{m_{k+1}}} & M_{p}^{p}\left(f_{n}, r\right) v(r) d \nu(r) \\
\leq & M_{p}^{p}\left(f_{n}, r_{m_{n}}\right) v\left(r_{m_{n}}\right) \int_{r_{m_{k}}}^{r_{m_{k+1}}}\left(\frac{r}{r_{m_{n}}}\right)^{m_{n}} \frac{v(r)}{v\left(r_{m_{n}}\right)} d \nu(r) \\
\leq & c_{0} M_{p}^{p}\left(f_{n}, r_{m_{n}}\right) v\left(r_{m_{n}}\right) I_{n}\left(\prod_{j=k}^{n-1} \frac{I_{j}}{I_{j+1}}\right)\left(\frac{1}{b}\right)^{|n-k|} \\
\leq & c_{1}\left(\frac{\rho}{b}\right)^{|n-k|} M_{p}^{p}\left(f_{n}, r_{m_{n}}\right) v\left(r_{m_{n}}\right) I_{n},
\end{aligned}
$$

where $c_{0}, c_{1}$ are universal constants. If $k \geq n$, then we use Lemmas 3.6(ii) and 3.7 (ii) to get

$$
\begin{aligned}
\int_{r_{m_{k}}}^{r_{m_{k+1}}} & M_{p}^{p}\left(f_{n}, r\right) v(r) d \nu(r) \\
\leq & M_{p}^{p}\left(f_{n}, r_{m_{n}}\right) v\left(r_{m_{n}}\right) \int_{r_{m_{k}}}^{r_{m_{k+1}}}\left(\frac{r}{r_{m_{n}}}\right)^{m_{n+1}} \frac{v(r)}{v\left(r_{m_{n}}\right)} d \nu(r) \\
\leq & K b M_{p}^{p}\left(f_{n}, r_{m_{n}}\right) v\left(r_{m_{n}}\right) I_{n}\left(\prod_{j=n}^{k-1} \frac{I_{j+1}}{I_{j}}\right)\left(\frac{1}{b}\right)^{|n-k|} \\
\leq & c_{2}\left(\frac{\rho}{b}\right)^{|n-k|} M_{p}^{p}\left(f_{n}, r_{m_{n}}\right) v\left(r_{m_{n}}\right) I_{n},
\end{aligned}
$$

where $c_{2}$ is a universal constant.

Conclusion of the proof of Theorem 3.1. Let $f \in A_{\mu}^{p}$, say, $f=\sum_{n} f_{n}$, where $f_{n}$ is as in Corollary 3.8. We can assume that $f_{n}=0$ for $n \leq k_{0}$ with $k_{0}$ as in (3.4).

To prove the right-hand inequality in Theorem 3.1 we use that $M_{p}\left(f_{n}, r\right) \leq$ $c M_{p}(f, r)$ for a universal constant independent of $r$, as well as that, in view of (3.1), $r^{m_{n}} v(r)$ is decreasing for $r \geq r_{m_{n}}$. We have

$$
\begin{aligned}
\sum_{n} & M_{p}^{p}\left(f_{n}, r_{m_{n}}\right) v\left(r_{m_{n}}\right) I_{n} \\
& \leq \sum_{n} \int_{r_{m_{n}}}^{r_{m_{n+1}}}\left(\frac{r_{m_{n}}}{r}\right)^{m_{n}} \frac{v\left(r_{m_{n}}\right)}{v(r)} M_{p}^{p}\left(f_{n}, r\right) v(r) d \nu(r) \\
& \leq \sum_{n} \int_{r_{m_{n}}}^{r_{m_{n+1}}}\left(\frac{r_{m_{n}}}{r_{m_{n+1}}}\right)^{m_{n}} \frac{v\left(r_{m_{n}}\right)}{v\left(r_{m_{n+1}}\right)} M_{p}^{p}\left(f_{n}, r\right) v(r) d \nu(r)
\end{aligned}
$$




$$
\begin{aligned}
& \leq K \sum_{n} \int_{r_{m_{n}}}^{r_{m_{n+1}}} M_{p}^{p}\left(f_{n}, r\right) v(r) d \nu(r) \\
& \leq c^{p} K \sum_{n} \int_{r_{m_{n}}}^{r_{m_{n+1}}} M_{p}^{p}(f, r) v(r) d \nu(r) \\
& \leq c^{p} K\|f\|_{p}^{p} .
\end{aligned}
$$

This in particular implies that $\sum_{n} M_{p}^{p}\left(f_{n}, r_{m_{n}}\right) v\left(r_{m_{n}}\right) I_{n}<\infty$.

Now we show the left-hand inequality of Theorem 3.1. Using the Minkowski inequality in the first estimate and Corollary 3.8 in the second one, we obtain

$$
\begin{aligned}
\|f\|_{p}^{p} & =\sum_{k} \int_{r_{m_{k}}}^{r_{m_{k+1}}} M_{p}^{p}(f, r) v(r) d \nu(r) \\
& \leq \sum_{k}\left(\sum_{n}\left(\int_{r_{m_{k}}}^{r_{m_{k+1}}} M_{p}^{p}\left(f_{n}, r\right) v(r) d \nu(r)\right)^{1 / p}\right)^{p} \\
& \leq c_{1} \sum_{k}\left(\sum_{n}\left(\frac{\rho}{b}\right)^{|n-k| / p}\left(M_{p}^{p}\left(f_{n}, r_{m_{n}}\right) v\left(r_{m_{n}}\right) I_{n}\right)^{1 / p}\right)^{p} \\
& \leq c_{2} \sum_{k} \sum_{n}\left(\frac{\rho}{b}\right)^{|n-k| / p} M_{p}^{p}\left(f_{n}, r_{m_{n}}\right) v\left(r_{m_{n}}\right) I_{n} \\
& \leq c_{3} \sum_{n} M_{p}^{p}\left(f_{n}, r_{m_{n}}\right) v\left(r_{m_{n}}\right) I_{n} .
\end{aligned}
$$

Here $c_{1}, c_{2}, c_{3}$ are universal constants. In the second to last inequality we used the Hölder inequality in the following way. Put $a_{n}=\left(M_{p}^{p}\left(f_{n}, r_{m_{n}}\right) v\left(r_{m_{n}}\right) I_{n}\right)^{1 / p}$. Then

$$
\sum_{n}\left(\frac{\rho}{b}\right)^{|n-k| / p} a_{n} \leq\left(\sum_{n}\left(\frac{\rho}{b}\right)^{|n-k| / p} a_{n}^{p}\right)^{1 / p} \cdot\left(\sum_{n}\left(\frac{\rho}{b}\right)^{|n-k| / p}\right)^{1 / q},
$$

with $1 / p+1 / q=1$. In the last inequality we interchanged the summation over $k$ and $n$ and utilized $\sup _{k} \sum_{n}(\rho / b)^{|n-k| / p}=\sup _{n} \sum_{k}(\rho / b)^{|n-k| / p}<\infty$.

\section{Solid Bergman spaces}

Recall that a Bergman space $A_{\mu}^{p}$ is solid if $S\left(A_{\mu}^{p}\right)=A_{\mu}^{p}$.

Theorem 4.1. Let $1<p<\infty, p \neq 2$. Then the following are equivalent:

(i) $A_{\mu}^{p}$ is solid,

(ii) $s\left(A_{\mu}^{p}\right)=A_{\mu}^{p}$,

(iii) the monomials $\left(z^{n}\right)_{n=0}^{\infty}$ are an unconditional basis of $A_{\mu}^{p}$,

(iv) the normalized monomials $\left(z^{n} /\left\|z^{n}\right\|_{p}\right)_{n=0}^{\infty}$ are equivalent to the unit vector basis of $l^{p}$,

(v) $\sup _{n}\left(l_{n+1}-l_{n}\right)<\infty$ for the numbers $l_{n}$ in (2.1).

Remark 4.2. If $p=2$, then the normalized monomials are an orthonormal basis for $A_{\mu}^{2}$ and all conditions (i)-(iv) are satisfied.

The following example is relevant in connection with Theorem 4.1. 
Example 4.3. Consider $R=\infty$ and $v(r)=\exp \left(-(\log r)^{2}\right), d \nu(r)=d r$. (This is included in Example 2.2 of [9].) Note that $v$ is decreasing on [1, $\infty$ [ which suffices in view of the remarks at the beginning of Section 3. We easily see that $r_{m}=\exp (m / 2)$ is the only zero of the derivative of $r^{m} v(r)$. Hence (3.1) is satisfied. We get, for any $n>0$ and $m>0$,

$$
\left(\frac{r_{m}}{r_{n}}\right)^{m} \frac{v\left(r_{m}\right)}{v\left(r_{n}\right)}=\left(\frac{r_{n}}{r_{m}}\right)^{n} \frac{v\left(r_{n}\right)}{v\left(r_{m}\right)}=\exp \left(\frac{(n-m)^{2}}{4}\right) .
$$

So, if we take $m_{n}=4 n$, then condition $\left(b_{0}\right)$ is satisfied with $b=e^{4}$. Moreover, we have $I_{n}=\exp (2 n+2)-\exp (2 n)$. An easy calculation shows that (3.2) holds. Hence we can consider $(2.1)$ with $l_{n}=m_{n} / p$. Therefore, $\sup _{n}\left(l_{n+1}-l_{n}\right)=4 / p<\infty$. This means that, for $d \mu(r)=v(r) d r$, the Bergman space $A_{\mu}^{p}$ is solid.

For the preceding example it is essential that $R=\infty$. Indeed, we have the following.

Corollary 4.4. Let $1<p<\infty, p \neq 2$, and $R=1$. Then no Bergman space $A_{\mu}^{p}$ is solid.

We prove Corollary 4.4 at the end of this section. For the proof of Theorem 4.1 we need the following.

Lemma 4.5. Let $\left(e_{n}\right)$ be a Schauder basis of a Banach space $X$ with basis projections $P_{n}$. For $M \subset \mathbb{N}$, let $T_{M}$ be the linear (not necessarily continuous) operator defined in the linear span of $\left(e_{n}\right)$ by $T_{M} e_{k}=e_{k}$ if $k \in M$ and $T_{M} e_{k}=0$ otherwise. If the basis $\left(e_{n}\right)$ is not unconditional, then there is $N \subset \mathbb{N}$ such that, for any $n$, there exists $m_{n}$ and $0 \neq y \in P_{m_{n}} X$ with $n\|y\| \leq\left\|T_{N} y\right\|$.

Proof. If $\left(e_{n}\right)$ is a conditional basis, then there exists an operator of the form $T_{N}$ which is unbounded on $X$. Hence there is a sequence $x_{k} \in X$ with $\left\|x_{k}\right\|=1$ and $\lim _{k \rightarrow \infty}\left\|T_{N} x_{k}\right\|=\infty$, and we find $k_{n}$ with $n=n\left\|x_{k_{n}}\right\|<\left\|T_{N} x_{k_{n}}\right\|$ for all $n$. Using $T_{N} P_{l}=P_{l} T_{N}$ for all $l$, we find $m_{n}$ such that

$$
0<n\left\|P_{m_{n}} x_{k_{n}}\right\| \leq\left\|P_{m_{n}} T_{N} x_{k_{n}}\right\|=\left\|T_{N} P_{m_{n}} x_{k_{n}}\right\| \text { for all } n .
$$
$\left(z^{n}\right)$.

In the following we retain the definition of $T_{N}$ with respect to the monomials

Lemma 4.6. Let $1<p<\infty, p \neq 2$, and assume that there are constants $c_{n}>0$, $d_{n}>0$ with $\sup _{n} d_{n} / c_{n}<\infty$, integers $0<a_{n}<b_{n}<a_{n+1}$, and radii $s_{n}$ such that, for any $f_{n} \in A_{\mu}^{p}$ with $f_{n}(z)=\sum_{a_{n} \leq j \leq b_{n}} \alpha_{j} z^{j}$, we have

$$
c_{n} M_{p}\left(f_{n}, s_{n}\right) \leq\left\|f_{n}\right\|_{p} \leq d_{n} M_{p}\left(f_{n}, s_{n}\right) .
$$

If $\sup _{n}\left(b_{n}-a_{n}\right)=\infty$, then the monomials are not unconditional in $A_{\mu}^{p}$.

Proof. It is well known that the monomials are a conditional basis sequence with respect to the norm $M_{p}(\cdot, 1)$. So we find $N \subset \mathbb{N}$ and $y_{n} \in Y_{n}:=\operatorname{span}\left\{z^{j}: 0 \leq\right.$ $\left.j \leq m_{n}\right\}$ with $M_{p}\left(y_{n}, 1\right)=1$ and $n \leq M_{p}\left(T_{N} y_{n}, 1\right)$. Find $k_{n}$ with $b_{k_{n}}-a_{k_{n}}>m_{n}$, put $Y_{n}=\left\{z^{j}: a_{k_{n}} \leq j \leq b_{k_{n}}\right\} \subset A_{\mu}^{p}$, and define $S_{n}: X_{n} \rightarrow Y_{n}$ by

$$
\left(S_{n} f\right)(z)=z^{a_{k_{n}}} f\left(z / s_{n}\right) \text {. }
$$


Then, according to our assumptions, we have $\left\|S_{n}\right\| \cdot\left\|S_{n}^{-1}\right\| \leq d_{n} / c_{n}<c$ for some universal constant $c$. Put $M_{n}=\left\{a_{k_{n}}+j: j \in N, j \leq m_{n}\right\}$. Then $S_{n} T_{N} S_{n}^{-1}=$ $\left.T_{M_{n}}\right|_{X_{n}}$. If we consider $M=\bigcup_{n} M_{n}$, then the preceding shows that $T_{M}$ is unbounded on $A_{\mu}^{p}$. This proves that the system of monomials is conditional in $A_{\mu}^{p}$.

Conclusion of the proof of Theorem 4.1. We have that (i) $\Leftrightarrow$ (ii) follows from the definition of solid hull, while (ii) $\Leftrightarrow$ (iii) follows from the definition of solid core. (Recall that, in any case, the monomials are a basis of $A_{\mu}^{p}$.) Now (iii) and Lemma 4.6 imply (v). Finally, (v) and (2.1) imply (iv), while (iv) trivially implies (iii).

Proof of Corollary 4.4. Proposition 3.5 of [8] shows that, for $R=1$, the assumptions of Lemma 4.6 are always satisfied. Hence the system of monomials can never be unconditional. In view of Theorem 4.1, the Bergman space $A_{\mu}^{p}$ can never be solid.

\section{Solid weighted spaces of entire functions with sup-norms}

In this section we consider weighted Banach spaces of analytic functions with sup-norms. The main result of this section, Theorem 5.2, complements Theorem 4.1. This result was announced in Remark 5.6 of [1]. Here, as in Section 3, a continuous weight $v: \mathbb{C} \rightarrow] 0, \infty[$ is a function satisfying

$$
\begin{aligned}
v(z) & =v(|z|), \quad z \in \mathbb{C}, \quad v(r) \geq v(s) \quad \text { if } 0 \leq r<s \quad \text { and } \\
\lim _{r \rightarrow \infty} r^{n} v(r) & =0 \quad \text { for all } n \geq 0 .
\end{aligned}
$$

We deal with the weighted space $H_{v}^{\infty}$ over $\mathbb{C}$, that is,

$$
H_{v}^{\infty}=\left\{f: \mathbb{C} \rightarrow \mathbb{C}: f \text { holomorphic, }\|f\|_{v}:=\sup _{z \in \mathbb{C}}|f(z)| v(z)<\infty\right\} .
$$

Let $H_{v}^{0}$ be the closure of the polynomials in $H_{v}^{\infty}$.

Similarly to the weighted $L_{p}$-norms in Sections 3 and 4 , one sees that it suffices to require only that $v(r) \geq v(s)$ for $r_{0} \leq r<s$ and some $r_{0}>0$, since $\|f\|_{v}$ and $\sup _{r_{0} \leq|z|<\infty}|f(z)| v(z)$ are equivalent for holomorphic $f$. Again, for $n>0$ let $r_{n} \in\left[0, \infty\left[\right.\right.$ be a point where the function $r \mapsto r^{n} v(r)$ attains its global maximum. The next lemma can be easily proved with induction (which was done in Lemma 5.1 of [9]). The indices $m_{n}$ are needed in the following.

Lemma 5.1. For any $b>2$ there are numbers $0<m_{1}<m_{2}<\cdots$ with $\lim _{n \rightarrow \infty} m_{n}=\infty$ and

$$
b=\min \left(\left(\frac{r_{m_{n}}}{r_{m_{n+1}}}\right)^{m_{n}} \frac{v\left(r_{m_{n}}\right)}{v\left(r_{m_{n+1}}\right)},\left(\frac{r_{m_{n+1}}}{r_{m_{n}}}\right)^{m_{n+1}} \frac{v\left(r_{m_{n+1}}\right)}{v\left(r_{m_{n}}\right)}\right) .
$$

Actually, one can show that Lemma 5.1 works for all $b>1$, but we need $b>2$ in the following proof. There are examples of weights on $\mathbb{C}$ such that the monomials $\left(z^{n}\right)_{n=0}^{\infty}$ are a Schauder basis in the Banach space $H_{v}^{0}$. This is the same as saying that the Taylor series of each element in $H_{v}^{0}$ converges with respect to the weighted sup-norm $\|\cdot\|_{v}$. In the known examples, in this case, $\left(z^{n} /\left\|z^{n}\right\|_{v}\right)_{n=0}^{\infty}$ is equivalent to the unit vector basis of $c_{0}$. Moreover, here $H_{v}^{\infty}$ is solid. We show 
that this is always true provided that $\left(z^{n}\right)_{n=0}^{\infty}$ is a Schauder basis of $H_{v}^{0}$. We also characterize this situation by a property for the indices $m_{n}$ of Lemma 5.1. Our arguments are similar to those of [8].

Let $h(z)=\sum_{k=0}^{\infty} b_{k} z^{k}$. As before, let $P_{n}$ be the partial sum operators, that is,

$$
\left(P_{n} h\right)(z)=\sum_{k=0}^{n} b_{k} z^{k}
$$

If the monomials are a basis of $H_{v}^{0}$, then $\sup _{n}\left\|\left.P_{n}\right|_{H_{v}^{0}}\right\|=\sup _{n}\left\|\left.P_{n}\right|_{H_{v}^{\infty}}\right\|<\infty$. For any $k$, we have

$$
\left|b_{k}\right| \cdot\left\|z^{k}\right\|_{v}=\left|b_{k}\right| r_{k}^{k} v\left(r_{k}\right)=\left|\frac{1}{2 \pi} \int_{0}^{2 \pi} h\left(r_{k} e^{i \varphi}\right) e^{-i k \varphi} d \varphi\right| v\left(r_{k}\right) \leq\|h\|_{v} .
$$

Moreover, take the numbers $m_{n}$ of Lemma 5.1 and put

$$
\left(R_{n} h\right)(z)=\sum_{k=0}^{m_{n-1}} b_{k} z^{k}+\sum_{m_{n-1}<k \leq m_{n}} \frac{\left[m_{n}\right]-k}{\left[m_{n}\right]-\left[m_{n-1}\right]} b_{k} z^{k} .
$$

Finally, put $M_{\infty}(h, r)=\sup _{|z|=r}|h(z)|$.

Theorem 5.2. The following are equivalent:

(i) $\sup _{n}\left(m_{n+1}-m_{n}\right)<\infty$ where $m_{n}$ are the indices of Lemma 5.1,

(ii) $\left(z^{n}\right)_{n=0}^{\infty}$ is a Schauder basis of $H_{v}^{0}$,

(iii) $\left(z^{n} /\left\|z^{n}\right\|_{v}\right)_{n=0}^{\infty}$ is equivalent to the unit vector basis of $c_{0}$,

(iv) $H_{v}^{\infty}$ is solid,

(v) $H_{v}^{0}$ is solid.

Proof. Put $V_{n}=R_{n}-R_{n-1}$. According to Proposition 5.2 in [9], since we assumed that $b>2$ in Lemma 5.1, the norms $\|h\|_{v}$ and $\sup _{n} \sup _{r_{m_{n-1}} \leq r \leq r_{m_{n+1}}} M_{\infty}\left(V_{n} h\right.$, $r) v(r)$ are equivalent. Since Lemma 3.3 in [9] implies that the operators $V_{n}$ are uniformly bounded on $H_{v}^{\infty}$, we obtain constants $c_{1}>0$ and $c_{2}>0$ with

$$
c_{1} \sup _{n}\left\|V_{n} h\right\|_{v} \leq\|h\|_{v} \leq c_{2}\left\|V_{n} h\right\|_{v} \quad \text { for all } h \in H_{v}^{\infty} .
$$

(i) $\Rightarrow$ (ii): Observe that, by the definition of $V_{n}$, $\operatorname{dim} V_{n}\left(H_{v}^{0}\right)=\left[m_{n+1}\right]-\left[m_{n-1}\right]$. By (i) we obtain $\sup _{n} \operatorname{dim} V_{n}\left(H_{v}^{0}\right)<\infty$. With the definition of $P_{j}$ and $(5.1)$, we see that $\sup _{j, n}\left\|\left.P_{j}\right|_{V_{n}\left(H_{v}^{0}\right)}\right\| \leq \sup _{n}\left(\left[m_{n+1}\right]-\left[m_{n-1}\right]\right)<\infty$. With (5.2) and $P_{j} V_{n}=V_{n} P_{j}$ for all $j$ and $n$, we conclude that the projections $P_{j}$ are uniformly bounded. Hence $\left(z^{n}\right)_{n=0}^{\infty}$ is a Schauder basis of $H_{v}^{0}$.

(ii) $\Rightarrow$ (i): Assume that (ii) holds. By definition, $V_{n}\left(P_{m_{n+1}}-P_{m_{n-1}}\right)=V_{n}$. In view of the uniform boundedness of the $V_{n}$ and (5.2), we obtain constants $c_{1}^{\prime}>0$ and $c_{2}^{\prime}>0$ with

$$
c_{1}^{\prime} \sup _{n}\left\|\left(P_{m_{n+1}}-P_{m_{n}}\right) h\right\|_{v} \leq\|h\|_{v} \leq c_{2}^{\prime} \sup _{n}\left\|\left(P_{m_{n+1}}-P_{m_{n}}\right) h\right\|_{v}
$$

for all $h \in H_{v}^{\infty}$. Here the first inequality follows from the uniform boundedness of the $P_{n}$ in view of (ii), while the second inequality follows from (5.2). Let $t_{n} \in[0, R[$ 
be such that

$$
t_{n}=r_{m_{n}} \quad \text { if } b=\left(\frac{r_{m_{n+1}}}{r_{m_{n}}}\right)^{m_{n+1}} \frac{v\left(r_{m_{n+1}}\right)}{v\left(r_{m_{n}}\right)}
$$

and

$$
t_{n}=r_{m_{n+1}} \quad \text { if } b=\left(\frac{r_{m_{n}}}{r_{m_{n+1}}}\right)^{m_{n}} \frac{v\left(r_{m_{n}}\right)}{v\left(r_{m_{n+1}}\right)}
$$

in Lemma 5.1 Then Corollary 3.2(b) of [9] implies that

$$
\left\|\left(P_{m_{n+1}}-P_{m_{n}}\right) h\right\|_{v} \leq 2 b M_{\infty}\left(\left(P_{m_{n+1}}-P_{m_{n}}\right) h, t_{n}\right) v\left(t_{n}\right) .
$$

With (5.3) we obtain

$$
\begin{aligned}
d_{1} \sup _{n} & M_{\infty}\left(\left(P_{m_{n+1}}-P_{m_{n}}\right) h, t_{n}\right) v\left(t_{n}\right) \\
& \leq\|h\|_{v} \leq d_{2} \sup _{n} M_{\infty}\left(\left(P_{m_{n+1}}-P_{m_{n}}\right) h, t_{n}\right) v\left(t_{n}\right)
\end{aligned}
$$

for some constants $d_{1}>0, d_{2}>0$ and all $h \in H_{v}^{0}$.

It is well known that there are bounded holomorphic functions whose Taylor series do not converge with respect to $M_{\infty}(\cdot, 1)$. By going over to suitable Cesàro means if necessary, we see that, for each $n \in \mathbb{N}$, there is a polynomial $f$ of degree $N$ and an index $M \leq N$ such that

$$
M_{\infty}(f, 1)=1 \quad \text { but } n \leq M_{\infty}\left(P_{M} f, 1\right) .
$$

Proceeding by contradiction, assume that (i) does not hold, that is, $\sup _{n}\left(m_{n+1}-\right.$ $\left.m_{n}\right)=\infty$. Then we find $k$ with $\operatorname{dim}\left(P_{m_{k+1}}-P_{m_{k}}\right) H_{v}^{0}>N$. Put $h(z)=$ $z^{m_{k}} f(z) / v\left(t_{k}\right)$. Then, in view of (5.4), we obtain

$$
d_{1} \leq\|h\|_{v} \leq d_{2} \quad \text { and } \quad \frac{n}{d_{2}} \leq\left\|P_{M+m_{k}} h\right\|_{v} .
$$

This implies that the projections $P_{j}$ are not uniformly bounded, contradicting assumption (ii). This contradiction implies that $\sup _{n}\left(m_{n+1}-m_{n}\right)<\infty$, and we have checked that (ii) $\Rightarrow$ (i).

Moreover, if $\sup _{n}\left(m_{n+1}-m_{n}\right)<\infty$, then (5.4) easily implies that the normalized monomials are equivalent to the unit vector basis of $c_{0}$. Hence we have (ii) $\Rightarrow$ (iii). The implication (iii) $\Rightarrow$ (ii) is trivial.

(iii) $\Rightarrow$ (iv): By the preceding we know already that (iii) implies (ii) and hence (5.4). If $\sigma_{n}$ is the $n$th Cesàro mean and $h \in H_{v}^{\infty}$, then $\sigma_{n} h \in H_{v}^{0}$. We have $\sigma_{n} P_{j}=P_{j} \sigma_{n}$ for all $n$ and $j$. Moreover, $\left\|\sigma_{n} h\right\|_{v} \leq\|h\|_{v}$ and $\sup _{n}\left\|\sigma_{n} h\right\|_{v}=\|h\|_{v}$. This implies that (5.4) remains valid for all $h \in H_{v}^{\infty}$. This together with the fact that $\sup _{n}\left(m_{n+1}-m_{n}\right)<\infty$ shows that $H_{v}^{\infty}$ is solid.

(iv) $\Rightarrow$ (iii): This implication follows from Theorem 5.2 in [1]. (iv) $\Rightarrow$ (v): If $g \in S\left(H_{v}^{0}\right)$, then by definition and (iii),

$$
\lim _{n \rightarrow \infty} \hat{g}(n)\left\|z^{n}\right\|_{v}=0,
$$

which implies by (iii) that $g \in H_{v}^{0}$.

$(\mathrm{v}) \Rightarrow(\mathrm{iv})$ : If $g \in S\left(H_{v}^{\infty}\right)$, then by definition $\sigma_{n} g \in S\left(H_{v}^{0}\right)=H_{v}^{0}$ for all $n$. This implies that $g \in H_{v}^{\infty}$. 
(In [9], the second author showed that $v(r)=\exp \left(-(\log r)^{2}\right), R=\infty$, satisfies (ii) (and hence all assertions) of Theorem 5.2.)

Note that in the preceding proof we do not use the fact that our functions are defined on $\mathbb{C}$. The arguments work just as well for weighted spaces of holomorphic functions over the unit disk $\mathbb{D}$. However, in this case $\lim _{n \rightarrow \infty} r_{n}=1$ and this fact together with

$$
4<b^{2} \leq\left(\frac{r_{m_{n+1}}}{r_{m_{n}}}\right)^{m_{n+1}-m_{n}}
$$

implies that $\sup _{n}\left(m_{n+1}-m_{n}\right)=\infty$ (in view of Lemma 5.1, which remains true over $\mathbb{D}$ ). This means that in the case of holomorphic functions over $\mathbb{D}$ the preceding theorem is empty (cf. Corollary 5.3 in [1]).

Acknowledgments. Bonet's work was partially supported by the MTM201676647-P and GV Prometeo/2017/102 projects (Spain). Taskinen's work was partially supported by a research grant from the Faculty of Science of the University of Helsinki.

\section{References}

1. J. Bonet, W. Lusky, and J. Taskinen, Solid hulls and cores of weighted $H^{\infty}$-spaces, Rev. Mat. Complut. 31 (2018), no. 3, 781-804. Zbl 06946770. MR3847084. DOI 10.1007/ s13163-018-0265-6. 469, 473, 474, 476, 481, 483, 484

2. J. Bonet and J. Taskinen, Solid hulls of weighted Banach spaces of analytic functions on the unit disc with exponential weights, Ann. Acad. Sci. Fenn. Math. 43 (2018), no. 1, 521-530. Zbl 1398.46021. MR3753191. DOI 10.5186/aasfm.2018.4334. 469

3. J. Bonet and J. Taskinen, Solid hulls of weighted Banach spaces of entire functions, Rev. Mat. Iberoam. 34 (2018), no. 2, 593-608. Zbl 06897378. MR3809450. DOI 10.4171/RMI/ 996. 475

4. P. L. Duren, Theory of $H^{p}$-Spaces, Pure Appl. Math. 38, Academic Press, New York, 1970. Zbl 0215.20203. MR0268655. 470

5. A. Harutyunyan and W. Lusky, On $L_{1}$-subspaces of holomorphic functions, Studia Math. 198 (2010), no. 2, 157-175. Zbl 1201.46026. MR2640075. DOI 10.4064/sm198-2-4. 471

6. M. Jevtić, D. Vukotić, and M. Arsenović, Taylor Coefficients and Coefficient Multipliers of Hardy and Bergman-Type Spaces, RSME Springer Ser. 2, Springer, Cham, 2016. Zbl 1368.30001. MR3587910. DOI 10.1007/978-3-319-45644-7. 469

7. J. Lindenstrauss and L. Tzafriri, Classical Banach Spaces, I, Ergeb. Math. Grenzgeb. (3) 92, Springer, Berlin, 1977. Zbl 0362.46013. MR0500056. 471

8. W. Lusky, On the Fourier series of unbounded harmonic functions, J. Lond. Math. Soc. (2) 61 (2000), no. 2, 568-580. Zbl 0956.46019. MR1760680. DOI 10.1112/S0024610799008443. $477,481,482$

9. W. Lusky, On the isomorphism classes of weighted spaces of harmonic and holomorphic functions, Studia Math. 175 (2006), no. 1, 19-45. Zbl 1114.46020. MR2261698. DOI 10.4064/sm175-1-2. 473, 480, 481, 482, 483, 484

10. W. Lusky and J. Taskinen, Toeplitz operators on Bergman spaces and Hardy multipliers, Studia Math. 204 (2011), no. 2, 137-154. Zbl 1237.47034. MR2805536. DOI 10.4064/ sm204-2-3. 471

11. M. Mateljević and M. Pavlović, $L^{p}$-behaviour of the integral means of analytic functions, Studia Math. 77 (1984), no. 3, 219-237. Zbl 1188.30004. MR0745278. DOI 10.4064/ sm-77-3-219-237. 470

12. M. Pavlović, Mixed norm spaces of analytic and harmonic functions, I, Publ. Inst. Math. (Beograd) (N.S.) 40(54) (1986), 117-141. Zbl 0619.46022. MR0883941. 470 
13. M. Pavlović, Function Classes on the Unit Disc: An Introduction, De Gruyter Stud. Math. 52, De Gruyter, Berlin, 2014. Zbl 1296.30002. MR3154590. 469

14. P. Wojtaszczyk, Banach Spaces for Analysts, Cambridge Stud. Adv. Math. 25, Cambridge Univ. Press, Cambridge, 1991. Zbl 0724.46012. MR1144277. DOI 10.1017/ CBO9780511608735. 470

${ }^{1}$ Instituto Universitario de Matemática Pura y Aplicada IUMPA, Universitat Politècnica de València, E-46071 Valencia, Spain.

E-mail address: jbonet@mat.upv.es

${ }^{2}$ Institute of Mathematics, University of Paderborn, D-33098 Paderborn, GerMANY.

E-mail address: lusky@math.upb.de

${ }^{3}$ Department of Mathematics and Statistics, P.O. Box 68, University of HelSINKI, 00014 HeLsinki, Finland.

E-mail address: jari.taskinen@helsinki.fi 